\title{
Application of the plastic optical fibre in domestic multimedia networks
}

\author{
Javier Mateo, M. Ángeles Losada, Alicia López \\ GTF, i3A, Universidad de Zaragoza, María de Luna 1, 50018 Zaragoza, Spain \\ Tel: +34976762360,Fax: +34976 762111,e-mail: jmateo@unizar.es
}

\begin{abstract}
Plastic Optical Fibre (POF) constitutes an advantageous alternative medium to implement domestic data networks. In this proposal, we develop an in-home network prototype for distributing video, audio and data information based on large core step-index plastic optical fibres (SI-POF). The aim is to demonstrate a viable uncluttered application of the POFs where the up to date technologies converge in a homogeneous domestic system.
\end{abstract}

\section{INTRODUCTION}

Nowadays, the average user has many consumer-electronics devices at home: TVs, DVDs, audio equipments, telephones, decoders, PCs and also, multiple incoming signals providing different contents: voice, video, music, etc. However, although the consumer needs for multimedia entertainment can be fulfilled with the existing products and technologies, the combination of audio, video and data signals over the same physical medium (Triple-Play) still poses a problem from the user point of view because there is not a simple way to integrate all these contents and equipments $[1,2]$.

One of the obstacles to this integration is that equipment manufacturers keep their own standards which do not allow them to interact with other equipment. In addition, the cabling infrastructure in the houses is not prepared for the newest technologies, although it is improving for the recently built ones.

Triple-play solutions offered by service providers are both closed and costly, because they are individually designed for a single user. However, the present technology allows a convergence of the different services so that they can be enjoyed in a distributed, homogeneous and simple way inside the home. A minimum initial investment in the house to get a data-oriented communication infrastructure is necessary with the view of a future in which coaxial or telephone cables will not be needed.

In the last few years, the market of programs and equipment for contents storage and distribution is increasing considerably at the level of private users. These equipments offer interesting functionalities, such as the possibility of sharing multimedia contents at different locations inside the house. Although they have a reasonable price, its management is restricted to users with advanced knowledge.

Now, more providers are offering products such as pay-per-view or video-on-demand as the consumers want to be able to enjoy the multimedia contents in the time and location they choose, but their costs are still quite high. In addition, the equipments required are diverse, difficult to interconnect and will be soon obsolete when new functionalities are added or new standards imposed.

In conclusion, the objectives of the electronics equipment manufacturers and of the service providers are far from adapted to those of the consumers. However, the present or near future technologies will allow to satisfy the user needs at a minimum cost. There are a number of reliable transmission media and protocols for video or voice over IP are widely known. Thus, the actual situation is ideal for the advent of domestic networks, where standard communication equipment is managed by the users to distribute multimedia contents homogeneously in their houses with quality and at a reasonable cost. Therefore, an analysis of the different alternatives from the user point of view is necessary.

In this paper, the application of plastic optical fibres in domestic networks is evaluated in the context of the problem of convergence of Triple-Play as the medium to distribute multimedia entertainment contents inside the house. Different transmission media are compared to POFs, revealing that its many advantages place it in a competitive position relative to other more traditional media. Also, the state of the art of POF applications in communications is briefly described. Finally, we present the design of a prototype network for distributing video, audio and data information based on large core step-index plastic optical fibre (SI-POF). This network has been implemented in our laboratory and it is presently working to demonstrate how all the home information sources can converge within a single open homogeneous system.

The work reported in this paper was supported by the Spanish Ministry for Science and Technology under grant TEC2009-14718-C03-02. 


\section{POF AS PHYSICAL TRANSMISSION MEDIA}

The demand for broadband services such as video on-demand raises the need of a high bandwidth service. One of the requirements posed by consumers is that network installation and operation has to be easy and cheap, preferably "do-it-yourself". Home networks offer a wide possible range of transmission media: coaxial cables, twisted copper pair cables, power lines, wireless, etc, which complicate the interoperability between devices even more as each network is optimised for a particular set of services. A single broadband network would provide an efficient solution for present and future devices.

The best candidate for a do-it-yourself home network would be PLC, in the sense that power outlets are everywhere inside the house. Nevertheless, using PLC as the backbone will not provide enough bandwidth for some situations and has the electrical background noise and the dependence on the quality of the electrical infrastructure as major disadvantages.

The new wireless standard $802.11 \mathrm{n}$ provides full connectivity for home applications and enough data rate to support real-time applications. However, in old houses with concrete walls, the signal coverage can be limited to a single room. In addition, even if theoretically there should be enough bandwidth, we have experienced frequent signal interruptions unacceptable for this kind of real-time applications [3].

Ethernet copper or fibre media are two technologies that require relatively high installation expense in built homes. However, these approaches work well in new structured houses and are good solutions for the backbone of the house as they provide high bandwidth and reliability for data communications. We have compared standard RJ45 copper cables with POF. The advantages of the POF over the RJ45 copper cables are basically physical and aesthetical. In a home network the aim is to use a communication medium that causes a minimum environmental impact. Nowadays, RJ45 copper cable is very widely used in LANs but has a number of disadvantages when compared to POF: copper cable is thicker and less malleable than POF while small size and curvature resistance is imperative in this kind of network. Besides, electromagnetic radiation can interfere with the transmitted signal in copper cables, while electrical background noise does not affect optical wires. Therefore, optical fibre could be installed through the same conduits that provide electricity $[4,5]$.

POF combines the ease of installation benefit of copper networks with the transmission advantages of conventional glass fibre (GOF). It also overcomes the problems that can arise with GOF which is too brittle and more difficult to handle. The simplicity of installation of POF combined with the added flexibility of the POF cable (down to a radius of $25 \mathrm{~mm}$ ) make it ideal for narrow cable shafts where installing POF would take shorter time than installing conventional thicker copper cable [6]. There are three more reasons to use POF as the network backbone. First, the PMMA fibre works best with visible light, mainly red, and thus hazard exposure can be easily avoided. Note that this advantage is lost when perfluorinated fibre is used as it works with infrared sources. Second, it is impossible to get electric shock using POF cables unlike with copper cables. Finally, the extensive use of optical fibres is steadily lowering the prices of connector and adapters needed for home installation. However, future gigabit requirements imply the use of graded index POF with less modal dispersion. Large core PMMA fibres keep the advantages of step index POFs, but it is difficult to find commercial optical transceivers working at $650 \mathrm{~nm}$ and gigabit rates. On the other hand, perfluorinated POFs are compatible with multimode GOF fibre and thus, can be used with commercial infrared transceivers. However, the advantage of lower hazard exposure is lost with infrared sources, and in addition, this fibre is thinner and not so easy to connect. Moreover, measurements of the bit error rate as a function of data rate reveal that even the large core step-index POF are suitable to carry Gigabit Ethernet for distances up to 25 meters, which are more than enough for most domestic networks [7].

Although there is still an uncertain future for POFs penetration into the home in Europe, it is already widely used in other countries, mainly in Asia. For example, the GigaHouse Town in a project designed by Keio University in Japan to demonstrated the viability and economic efficiency of a city with highly computerized homes to contribute to bring the information technology to the average citizen [8]. Also, the Information Ministry of South Korea has a proposal for cabling homes using POF [9]. In the USA, the Plastic Optical Fibre Trade Organization (POFTO) is promoting industry awareness, acceptance, and advancement of plastic optical fibre and component systems in the data communications markets and promoting the advancement and acceptance of standards incorporating POF [10]. In Europe, from the beginning of 2006, the POF-ALL project and its continuation, POF-PLUS, have gathered some European research centres and companies with the purpose of developing a low-cost solution based on POF to make the delivery of broadband access to everyone possible $[11,12]$. Over the last year, a considerable advance in the production of standard POF commercially available devices has been also made by European companies such as Firecomms Ltd.[13], Homefibre Digital Network GmbH [14], Diemount GmbH [15], Luceat S.p.A. [16], etc. 


\section{DESIGN AND IMPLEMENTATION OF A POF-BASED DOMESTIC NETWORK}

In our configuration, all the home information sources converge within a single homogeneous open system. This system is based on standard equipment, devices and protocols allowing scalability and accommodation of future trends. Moreover, costs are kept as low as possible since this application is addressed to a domestic market.

\subsection{Network}

The system is conceived as a hierarchical structure based on a server and several clients interconnected in a Fast Ethernet network. In our network the preferable topology would be a daisy chain or a tree in order to reduce the installation cost and the aesthetical impact over built houses. The backbone of the network is made by SI-POF operated at a visible wavelength while the connection to the electronic devices is made by standard RJ 45 copper cables. A typical layout of this network is shown in Figure 1.

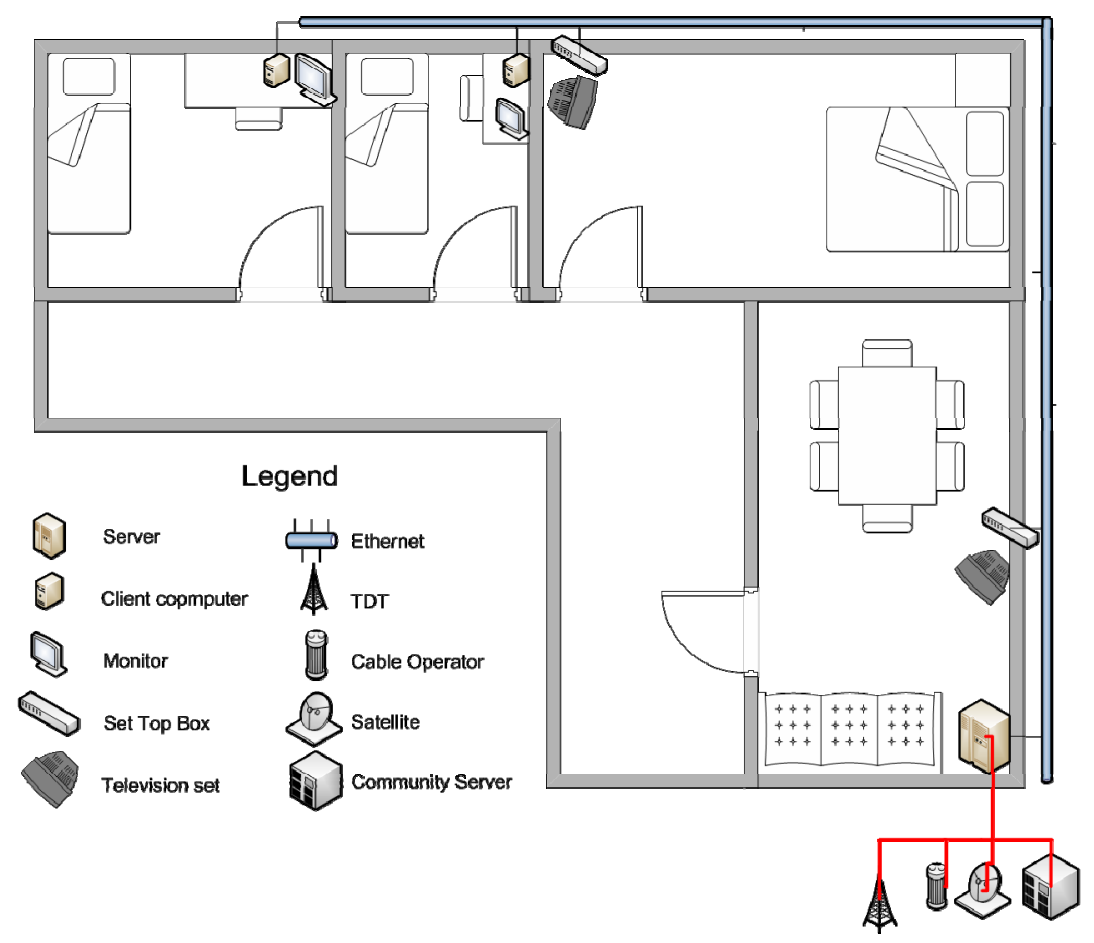

Figure 1. Example of a domestic network.

The media conversion is made by low-cost transceivers or electro-optical switches. The physical transmission of the implemented network is based on standard 1-mm SI-POF without connectors by using Optospider 650-nm SFP duplex and simplex optoelectronics modules from Diemount, RJ45 and SFP mixed switches (CS-Fx2) from the Taiwanese company Wamin [17], and Firecomms POF-Fast Ethernet MCE300T-200 converters. Although the principal transmission medium in our network is POF, we have also introduced a WiFi router to allow wireless connection. In addition, we have used copper cables for some short connections because it is the typical connection medium for consumer electronics and is massively distributed at home. Thus, we can compare the performance of the three transmission media for audio, video and data signals. Figure 2 shows the commercial devices that compose the implemented network.

A POF-Fast Ethernet converter is connected to the server Ethernet card, and from there to the first switch we use POF and a SFP duplex. We have used RJ45 and SFP mixed switches, thus we use RJ45 copper cables for connecting close clients and SFP simplex and POF for connecting the next far switch. At this switch, we also use copper cable for close clients and POF for longer distances.

The system uses mainly free open source software (Videolan VLC) or cheap shareware developments (DVBViewer and its accessories, DVBServer, and HTTPServer). VLC and DVBViewer complement each other and can support different client types. We have configured the programs and adapted them to meet the required objectives. 


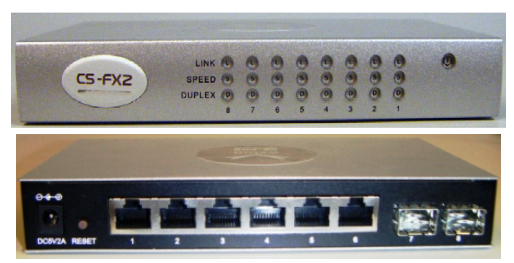

a) Switch SFP CS-FX2.

Front and rear views

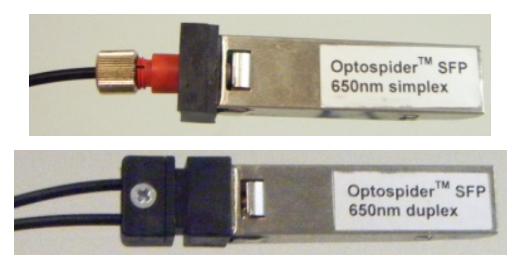

b) Optospider 650- $\mathrm{nm}$ SFP. Simplex and duplex

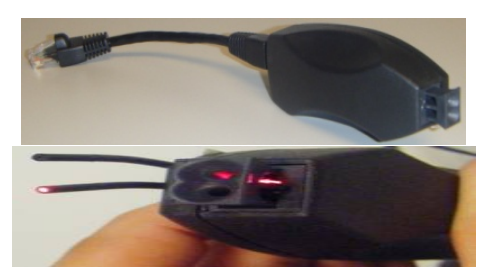

c) Firecomms POF Fast Ethernet converter

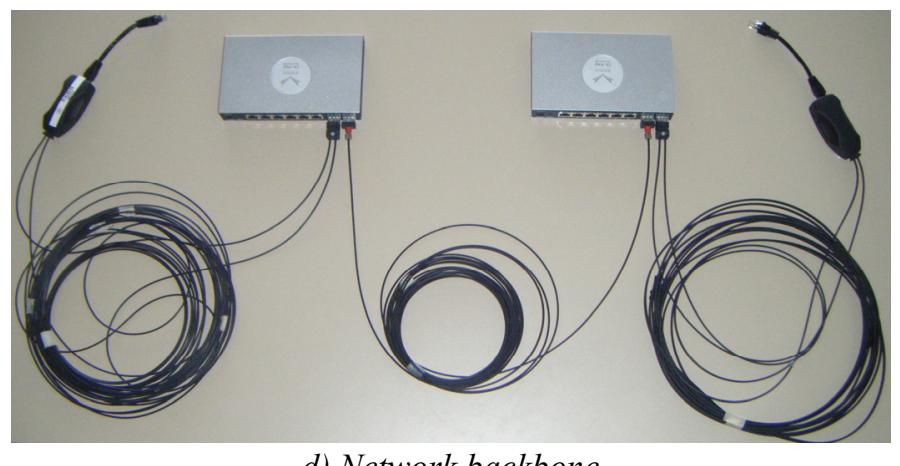

d) Network backbone

Figure 2. Devices and backbone of the implemented network.

\subsection{Server and clients}

Nowadays, the best cost-effective server can be implemented on a standard PC platform. In our system, the server is able to record simultaneously multiple live contents on demand and can also follow a programmable schedule. Moreover, the server stores films, music, pictures and live recordings to serve them later to each client on demand. The technical requirements to be a server-PC in our system are lower than those of a modern desktop PC that, in addition, can act itself as a client.

The number of USB decoders needed to connect the server to the TDT signal depends on the number of transponders that we want the system to be able to access simultaneously. In order to avoid restrictions, the system should have as many decoders as TDT transponders. We concluded, however, that a good compromise is reached if we use only one decoder per client. In this way, the system will be cheaper than in the first design but it still will be able to meet all the requirements. As it is not frequent to have all the clients working simultaneously in this type of application, there will always remain some unused decoders that the server can use for recording.

The clients can be multiplatform, depending on the user needs. In our prototype, which is shown in Figure 3, we adopted and configured desktop PCs, Set Top Boxes (Amino [18]) and Pocket PCs as different clients. The Set Top Boxes are less versatile than PCs because they are designed only for this particular multimedia application, while on the other hand PCs have many other applications. Therefore, having tested both types of client in practice, and as they have a similar price nowadays, we recommend the use of a PC in any home dependence. Each client can display any of the TDT channels in real time or any of the files stored on the server. Moreover, the users can easily program recordings from the client to be stored either on this same client or on the server. The client management is user-friendly and can be achieved through an infrared remote control.

Finally, the rise of so many television standards (HD ready, HD TV, Full HD, etc) has confused the average user. For example, many people acquire HD ready televisions because of their lower prize relative to the other standards, only to realize that the HD ready televisions are incapable of decoding HD television signals. Instead, our implemented system has not this type of limitation and it is capable of decoding HD television.

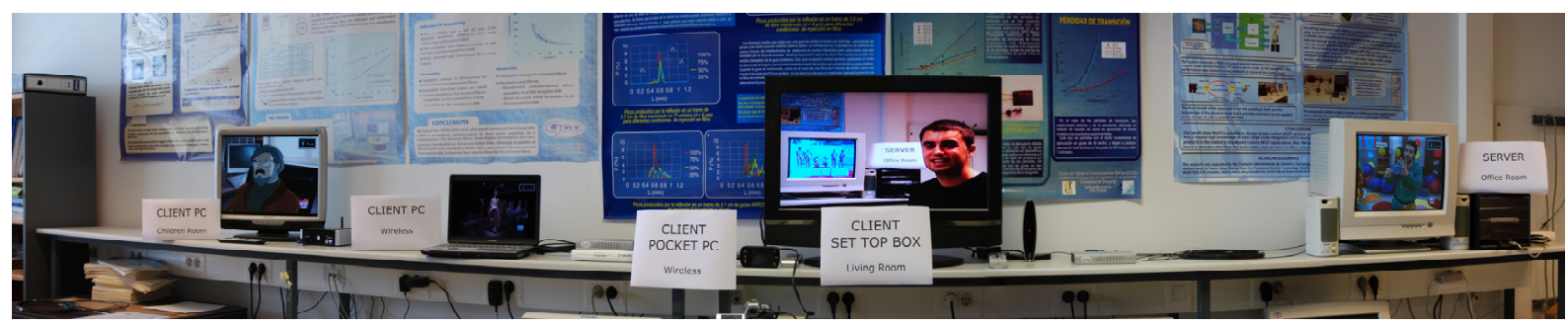

Figure 3. Network prototype as implemented in our laboratory. 


\section{CONCLUSIONS}

In this paper we have analyzed the scope of multimedia home applications and we have verified the proper behaviour of a multimedia home system prototype for distributing video, audio and data information, demonstrating how all the home information sources can converge within a single open homogeneous system. This system is conceived as a hierarchical structure based on a computer acting as the server and several clients interconnected in a Fast Ethernet network arranged in a daisy chain topology. The backbone of the network is SI-POF operated at a visible wavelength while the final end to the electronic devices is standard RJ45 copper cables, and the media conversion is performed by low-cost transceivers or electro-optical switches with several electrical ports to connect the different devices. Although wireless is the best-positioned technology as the transmission medium for houses, it does not reach the effective bandwidth requirements due to wall-material problems and thus, its reliability remains in question. Between the wired media, we have verified the ease of POF installation, which is its main advantage. First, only when using POF, a connection can be easily checked because it operates at a visible wavelength. Also, the hand-made network assembly is rendered simpler as the visible light facilitates the connection of the fibre with its components. Therefore, we can state that POF and its components are at least as easy to install as copper cable is. In addition, the flexibility of POF cable down to a small radius makes it ideal for narrow cable shafts where installing POF will be faster than installing conventional copper cable. Finally, we must emphasize that our system backbone is behaving correctly and that both the POF and the prototypes used in the implemented network (switches, convertors and connectors) have shown a high reliability.

\section{REFERENCES}

[1] J. Faller, "POF for structured Cabling in Homes and Small Business," Tutorial of the POF World West, Santa Clara, USA 2006.

[2] D. Cleevely, "Convergence in the Home," IET Communications Engineer, 2006, pp. 10-15.

[3] J. Mateo, A. Oca, M.A. Losada, J. Zubia, "Domestic multimedia network based on POF," in Proc. 17th International Conference on Polymer Optical Fibre and application, Santa Clara ,USA, August 2008.

[4] U. Schelinski, K. Frommhagen, M. Scholles, "A home networking infrastructure using POF based on IEEE 1394," in Proc. 13th International Conference on Polymer Optical Fibre and application, Nürnberg, Germany, September 2004, pp. 5966.

[5] O. Ziemann, H. Poisel, M Bloos, "POF on the way home," in Proc. 14th International Conference on Polymer Optical Fibre and application, Hong-Kong China, September 2005, pp. 351-355.

[6] B. Offenbeck, S. Junger, W. Tschekalinskij, N. Weber, "Possibilities and limitations of data communications over large core diameter PMMA POF," in Proc. of the 15th International Conference on Polymer Optical Fibre and application, Seoul, Korea, 2006, pp. 82-87.

[7] A. Lopez, P. Heredia, J. Mateo, M.A. Losada, "Comparative analysis of the transmission capabilities of large core plastic optical fibres," in Proc. ICTON-MW 2009, Angers, France, December 2009.

[8] Y. Koike, "Status of High-Speed Plastic Optical Fibre Towards Giga House Town," in Proc. of the 18th International Conference on Polymer Optical Fibre and application, Sydney, Australia, 2009.

[9] J.W. Lee, "Realization of FTTH for the integration of telecommunication and broadcasting," in Proc. 12th International Conference on Polymer Optical Fibre and application, Seattle, USA, September 2003, pp. 20-23.

[10] POFTO web page: http://www.pofto.org/

[11] D. Cárdenas, A. Nespola, S. Camatel, S. Abrate, and R. Gaudino, "100Mb/s Ethernet Transmission over 275m of Large Core Step Index Polymer Optical Fiber: Results from the POF-ALL European Project", IEEE Journal of Lightwave Technology, Volume 27, Issue 14, 2009 pp. 2908-2915, July 2009.

[12] S. Abrate, R. Gaudino, A. Antonino, S. Straullu, and P. Savio, "POF-PLUS project: toward Gbit/s transmission over SI-POF for home networking," in Proc. 17th International Conference on Polymer Optical Fibre and application, Sydney, Australia, September 2009.

[13] Firecomms Ltd. web page: http://www.firecomms.com/

[14] Homefibre Digital Network GmbH web page: http://homefibre.at/

[15] Diemount GmbH web page: http://www.diemount.com/

[16] Luceat S.p.A. web page: http://www.luceat.it/

[17] Wamin Optocomm web page: http://www.wamin.com.tw/

[18] Amino Technologies plc web page: http://www.aminocom.com/ 